\section{Does Mars have a magnetosphere?}

\section{from Alan Johnstone}

THE flow of magnetised plasma outward from the Sun, known as the solar wind, is supersonic with a Mach number of 6 to 8 in relation to planetary bodies it encounters. If it is deflected around a planet it forms a bow shock wave whose shape can be deduced by analogy with the flow of a gas around a suitably-shaped blunt body. Shock waves have been detected up-stream from Mercury, Venus, Earth, Mars and Jupiter but not the Moon because it absorbs all the plasma without deflecting it. For all these bodies, except Mars, the means by which the solar wind is deflected is known in principle if not in detail. Mercury, Earth and Jupiter deflect it magnetically, forming a magnetosphere whose boundary, or magnetopause, is found where the magnetic pressure exerted by the dipolar magnetic field balances the pressure of the solar wind. At Venus, the ionosphere forms a barrier to the flow as its magnetic field is too weak to deflect the solar wind before it encounters the atmosphere.

Mars remains controversial because although it has a thin atmosphere it is not known whether its intrinsic magnetic field is sufficiently strong to deflect the solar wind first. The only evidence for a Martian magnetic field comes from measurements made in the interaction region by three Russian spacecraft which orbited the planet. Russell (Geophys. Res. Lett. 5, 81; 5, 85 ; 1978) has criticised the interpretation of Dolginov (Geophys. Res. Lett. $5,89 ; 5,93 ; 1978)$ who claims that Mars has a magnetosphere. The Mars 2 and Mars 3 spacecraft crossed the shock wave on the dayside and approached the planet on the sunward side. Mars 5 crossed the shock wave near the terminator and then dipped into the magnetic tail or wake behind the planet. The different sets of data raise different issues and have been treated separately.

The argument over Mars 2 and Mars 3 initially concerns the identification of the shock wave. Knowing the Mach number of the flow and an approximate shape for the obstacle, the size of the obstacle can be deduced from the position of any shock traversal relative to the centre of Mars using the gas-dynamic analogy. If the

Alan Shnstone is at the Mullard Space Science Laboratory of University College, London. obstacle height is found to be more than $400 \mathrm{~km}$ or so above the surface of Mars then it is almost certainly magnetospheric. The dipole moment of Mars can then be calculated from the obstacle height and solar wind pressure. Dolginov places the shock wave at the point where the magnetic field first increases as the planet is approached (point 1 in the figure) and claims that between 2 and 3 the spacecraft is within the Martian magnetosphere. The shock front can also be detected in plasma measurements made by Bogdanov and Vaisberg (J. geophys. Res. 80, 487; 1975) and Gringauz et al. (J. geophys. Res. 81, 3349 ; 1976) by a broadening of the ion velocity distribution as the directed flow of the solar wind is thermalised in the shock. The location of the shock obtained this way lies close to the point identified as the magnetopause by Dolginov. The broadening of the ion velocity distribution is a necessary feature of the shock to allow for deflection of the flow and is therefore a more reliable indication. The magnetic field disturbance can be caused by waves propagating upstream from the shock. Russell uses the plasma data of Gringauz et al. to obtain a mean obstacle height of $400 \mathrm{~km}$. There is a great deal of scatter in the individual points.

It may be thought that the increase in magnetic field as the satellite

Mars 3 magnetogram of 21 January, 1972. The $X$ direction is towards the Sun. The $Z$ direction is towards the north pole of the ecliptic and the $Y$ direction completes a left-handed coordinate system. Circles 1 and 4 indicate bow shock crossings, 2 and $3-$ magnetopause crossings. The lower curve shows the ion flux from $0.3-$ $0.15 \mathrm{keV}$. (from Dolginov Geophys. Res. Lett op. cit.).

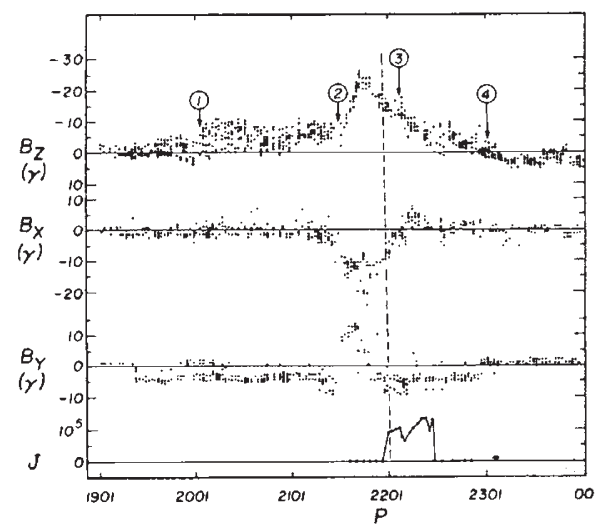

approaches Mars is a sure sign of an intrinsic magnetic field but there are two alternative explanations. First, as the solar wind passes through the shock it is compressed and the magnetic field is thereby increased. In pictorial terms, the field lines pile up as they encounter the obstacle, they become draped over its surface and eventually slip away to the side and drift downstream. Second, if there is sufficient conductivity in the Martian ionosphere, currents will be induced in the surface to prevent the penetration of solar wind field into the ionosphere. Russell argues that the configuration of the magnetic field between points 2 and 3 in the figure is what would be expeoted if the field lines were draped over the obstacle. Dolginov tries to fit these measurements to a dipolar field but the inconsistency of the results raises grave doubts about their significance.

Mars 5 provided more observations of the shock, and dipped into the wake behind the planet. In the wake region the plasma flow velocity is much lower and possibly isotropic and the magnetic field less variable than in the shocked solar wind flow. Dolginov argues that the region is part of the magnetic tail of the planet because the direction of the radial component stays the same even when the magnetic field in the solar wind reverses its direction. Russell points out that if the field in the tail was of Martian origin it should be predominantly radial whereas the measured field is more consistent with the configuration of field lines draped around the obstacle. Russell concludes that none of the probes has detected an intrinsic Martian magnetic field and that an upper limit to its possible value can be placed at $2 \times 10^{21}$ gauss $\mathrm{cm}^{3}$. Dolginov insists that both sets of data are consistent with a Martian dipole moment of $2.2 \times 10^{22}$ gauss $\mathrm{cm}^{3}$-an order of magnitude higher than Russell's limit.

The controversy will not be resolved until accurate plasma and magnetic field measurements are made nearer to the Martian surface. It is surprising to find that no plasma or magnetic field measurements have been made closer to the planet than $1,100 \mathrm{~km}$. If the solar wind is deflected by an obstacle at $400 \mathrm{~km}$ measurements are needed below this level to identify the nature of the interaction and establish the value of the Martian dipole moment. Even if the planet has a smaller dipole moment than Dolginov estimates, the magnetic field could still have an important role in the interaction and make the Martian ionosphere/magnetosphere system an especially interesting case to study. 